\title{
Surgical treatment of adrenal metastasis from renal cell carcinoma: a single-centre experience of 45 patients
}

\author{
ALESSANDRO ANTONELLI, ALBERTO COZZOLI, CLAUDIO SIMEONE, DANILO ZANI, TIZIANO ZANOTELLI, ELENA PORTESI \\ and SERGIO COSCIANI CUNICO \\ Department of Urology, University of Brescia, Brescia, Italy
}

Accepted for publication 1 September 2005

\section{OBJECTIVE}

To report, in a retrospective study, the diagnostic problems and oncological results of surgery in patients with either synchronous or metachronous adrenal metastasis, which are uncommon in renal cancer, at $2-10 \%$ of patients.

\section{PATIENTS AND METHODS}

Of 1179 patients treated for renal cancer between 1987 and 2003, 914 had renal surgery with concomitant ipsilateral adrenalectomy (routinely in 875 and for abnormal findings on computed tomography, $\mathrm{CT}$, in 39) and 15 contralateral adrenalectomy (all after suspicious findings on CT). During the follow-up after renal surgery, another 14 patients had adrenalectomy for CT evidence of an abnormal adrenal gland, contralateral to the previous renal tumour in 12 and bilaterally in two.

\section{RESULTS}

Of 914 ipsilateral adrenal glands removed during renal surgery, 854 (93.5\%) were normal on pathological examination, 28 (3\%) had a benign pathology, six (0.8\%) were directly infiltrated by the tumour and 26 $(2.7 \%)$ were metastatic. For both benign and metastatic ipsilateral adrenal pathology, CT had sensitivity, specificity and positive/ negative predictive values of 47\%, 99\%, 73\% and $96 \%$, respectively. Of 29 contralateral glands removed because of suspicious CT findings (15 at diagnosis of renal cancer, 14 during the follow-up) there was no abnormality in one (3.4\%), a benign pathology in seven (24\%) and a metastasis in 21 (72\%). Thus there were 32 synchronous (incidence 2.7\%; ipsilateral to the renal tumour in 24 , contralateral in six and bilateral in two), and 13 metachronous adrenal metastases (incidence 1.0\%; contralateral in 11 and bilateral in two). The metachronous metastases were diagnosed at a mean (range) interval of 30.6 (8-73) months after renal surgery. No ipsilateral adrenal metastases were discovered at diagnosis or during the follow-up in the 382 patients with an organconfined renal tumour of $<4 \mathrm{~cm}$ in diameter. Twenty-seven patients with an isolated adrenal metastasis (synchronous in 14, metachronous in 13) had statistically significantly $(P<0.001)$ better survival than the 18 (all synchronous) with multiple sites of metastatic disease. In particular, there was long-term survival (mean 83 months) in 10 patients with an isolated adrenal metastasis.

\section{CONCLUSION}

Sparing the ipsilateral adrenal is advisable only for organ-confined renal tumours of $<4 \mathrm{~cm}$ in diameter; clinical local staging of renal cancer is the best predictor of the risk of adrenal metastasis. Conversely, CT had good diagnostic ability for the contralateral adrenal gland, especially during the follow-up. Some patients with isolated adrenal metastasis could be treated by metastasectomy, with long-term survival free of disease and confirming that, even if in a few and unselectable patients, removing all the neoplastic bulk can be curative. Nevertheless, the high rate of relapse underlines the need for an effective systemic therapy, and more so for widespread metastatic disease that currently cannot be cured.

\section{KEYWORDS}

adrenal metastasis, renal cell carcinoma, metastasectomy, survival

\section{INTRODUCTION}

RCC can metastasize to practically all organs, following routes of spread and patterns that are not yet fully understood. Adrenal metastasis is a good example, as it can be synchronous or metachronous to the renal neoplasm, ipsilateral, contralateral or bilateral, solitary or part of a diffuse metastatic disease. In the present retrospective study we report the diagnostic problems and results of surgery in patients with either synchronous or metachronous adrenal metastasis.

\section{PATIENTS AND METHODS}

Between January 1987 and December 2003, 1179 consecutive patients with either uni(1136) or bilateral (43) RCC were treated surgically (497 women and 696 men; mean age 61.5 years, range 20.6-94.9), during 1193 surgical procedures. During the staging of the renal mass, CT detected some adrenal abnormalities (enlarged diameter, irregular morphology, heterogeneous density, contiguous infiltration by the neoplasm) in 48 patients, ipsilateral to the renal neoplasm in 33 , contralateral in nine and bilateral in six, giving 54 suspicious adrenal units.
A radical nephrectomy with concomitant ipsilateral adrenalectomy was performed when preoperative assessments showed an adrenal abnormality, or routinely if the renal tumour was ineligible for nephron-sparing surgery. However, in a few patients at high surgical risk or with contralateral adrenal disease, the sparing of the ipsilateral adrenal gland was preferred if no anomalies were apparent and if the renal neoplasm did not involve the upper pole. Ipsilateral adrenalectomy was not usual during elective or imperative nephron-sparing surgery, unless adrenal pathology was suspected and a metastasis was excluded by immediate histological examination. Table 1 
shows the procedures used with and with no ipsilateral adrenalectomy. A contralateral adrenalectomy was done only if there was a suspicion on preoperative imaging. Thus, an ipsilateral adrenalectomy accompanied renal surgery in 914 patients, 875 with normal and 39 with abnormal CT findings, and a contralateral adrenalectomy in 15, all with suspicious CT results.

All the patients treated for RCC in our department are monitored indefinitely by periodic clinical and radiological (chest X-ray, abdominal ultrasonography and/or CT) evaluations. The mean (range) follow-up for the entire group was 49.6 (12-252) months. During the follow-up, an adrenal mass with pathological features on CT (diameter $>3.5 \mathrm{~cm}$, growth with time, irregular margins, heterogeneous density, presence of calcifications, basal CT density $>10$ Hounsfield units, and rapid contrast medium wash-out) was detected in 14 asymptomatic patients. The lesion was contralateral to the side of previous kidney surgery in 12 patients and bilateral in the other two.

The RCC was staged using the TNM 2002 system and therefore cases with direct adrenal invasion (stage pT3a) were not considered as metastatic. The histological subtypes were all determined using the Heidelberg classification [1].

The Kaplan-Meier method was used for the survival analysis, with the log-rank test to compare survival rates between different groups (significant at $P<0.05$ ). The survival time was calculated from the diagnosis of adrenal metastasis for both synchronous and metachronous metastasis (i.e. excluding the interval between the diagnosis of kidney cancer and metastasis), so that we could compare groups of patients with oncological features (appearance of adrenal metastasis) that were as similar as possible.

\section{RESULTS}

Of 914 adrenal glands ipsilateral to RCC, 854 were normal on pathological examination (93.5\%, 844 with negative and 10 with positive preoperative CT), 28 had a benign pathology $(3.0 \%, 17$ with negative and 11 with positive CT findings), 26 had a metastasis (2.7\%, nine with negative and 17 with positive CT findings) and six had a neoplastic infiltration with a contiguous extension of RCC $(0.8 \%$, five with negative and one with

\begin{tabular}{|c|c|c|c|c|c|c|}
\hline \multirow[b]{2}{*}{ Stage } & \multicolumn{2}{|c|}{$\begin{array}{l}\text { No ipsilateral } \\
\text { adrenalectomy }\end{array}$} & \multicolumn{2}{|c|}{$\begin{array}{l}\text { Ipsilateral } \\
\text { adrenalectomy }\end{array}$} & \multirow[b]{2}{*}{ Total } & \multirow{2}{*}{$\begin{array}{l}\text { TABLE } 1 \\
\text { Surgical options and } \\
\text { pathological staging in }\end{array}$} \\
\hline & $\overline{N S S}$ & Nephrectomy & $\overline{N S S}$ & Nephrectomy & & \\
\hline I & 219 & 41 & 5 & 392 & 657 & 1193 surgical procedures \\
\hline$\|$ & 1 & 4 & 1 & 85 & 91 & for RCC \\
\hline III & 9 & 8 & 1 & 267 & 285 & \\
\hline IV & - & 3 & - & 157 & 159 & NSS, nephron-sparing \\
\hline Total & 229 & 56 & 7 & 901 & 1193 & surgery. \\
\hline
\end{tabular}

\begin{tabular}{|c|c|c|c|c|}
\hline Staging & Ipsilateral & Contralateral & Bilateral & \multirow{7}{*}{$\begin{array}{l}\text { Loco-regional staging of } \\
\text { kidney cancer in patients } \\
\text { with a synchronous adrenal } \\
\text { metastasis. }\end{array}$} \\
\hline \multicolumn{4}{|c|}{ Tumour limited to the renal capsule } & \\
\hline Diameter $<4 \mathrm{~cm}$ & 0 & 0 & 0 & \\
\hline Diameter $>4 \mathrm{~cm}$ & 2 & 2 & 0 & \\
\hline $\begin{array}{l}\text { Invasion of perirenal tissues } \\
\text { or venous involvement }\end{array}$ & 12 & 4 & 1 & \\
\hline Invasion of other organs & 2 & 0 & 1 & \\
\hline Lymph node metastasis & 8 & 0 & 0 & \\
\hline
\end{tabular}

positive $\mathrm{CT}$ ). Thus, for diagnosing the pathology (both benign and metastatic) of the ipsilateral adrenal gland, CT had a sensitivity, specificity, positive and negative predictive values of $47 \%, 99 \%, 73 \%$ and $96 \%$, respectively. Of 15 adrenal units removed contralateral to the renal surgery because of CT findings, there was no abnormality in one, benign pathology in six and a metastasis in eight.

\section{Overall, 32 patients had a synchronous} adrenal metastasis (21 men and 10 women, mean age 60 years, range 36-80), ipsilateral to the RCC in 24, contralateral in six and bilateral in two. In these patients the renal tumour affected the upper pole, either exclusively or with wider involvement of the organ, in $81 \%, 17 \%$ and all the ipsilateral, contralateral and bilateral cases, respectively; the respective mean (range) diameter of the renal neoplasm was 9.9 (5-20), 7.3 (4-9) and $9(8-10) \mathrm{cm}$, and that of the adrenal metastasis was 2.7 (0.5-9), 4 (1.5-7) and 5 (4-6) $\mathrm{cm}$. In the nine cases of ipsilateral metastasis with a negative preoperative CT, the mean diameter of the adrenal mass was $1.4(0.5-3.2) \mathrm{cm}$. The histological examination of the renal neoplasm showed conventional RCC in 30 patients (three with sarcomatoid aspects), i.e. $2.9 \%$ of the 1011 conventional RCC treated, and a papillary carcinoma in two, i.e. $2.7 \%$ of the 74 RCC of the same histology. The histological subtype of the adrenal metastasis was always concordant with that of the renal neoplasm.
Data on the loco-regional staging of renal cancer (i.e. without considering distant metastasis) in patients with synchronous metastasis are reported in Table 2: notably, in no patients was there a capsule-confined tumour of $<4 \mathrm{~cm}$ in diameter. The pathological findings of the 16 adrenal units detected by CT in 14 patients during the follow-up showed a benign pathology in one and a metastasis in 13 (eight men and five women, mean age 64 years, range 42-82), contralateral to the previous neoplasm in 11 and bilateral in two. The RCC was treated with nephroadrenalectomy in nine of the 13 patients, whereas an adrenal-sparing nephrectomy was used in the other four (including the two with bilateral metastases). At the time of renal surgery a conventional RCC, with a mean diameter of $9(1-17) \mathrm{cm}$, with no distant metastasis (stage I in three, stage III in 10) was diagnosed. The mean diameter of the metachronous adrenal metastasis was $3.6(1-6.5) \mathrm{cm}$. The median interval between treating RCC and the diagnosis of adrenal metastasis was 30.6 (8-73) months.

After complete staging (abdomen, thorax, brain $\mathrm{CT}$, and a bone scan), of the 32 patients with a synchronous adrenal metastasis, 14 had an isolated metastasis to the adrenal while the other 18 had multiple-site metastatic disease, eligible for surgical, medical and/or radiation therapy in eight and for only palliative therapy in 10. This group was monitored for a mean follow-up of 34.4 (12-148) months. 
FIG. 1. The Kaplan-Meier survival curves for the 27 patients whose disease was clinically confined to the adrenal gland (synchronous in 14 and metachronous in 13) and those with diffuse metastasis.

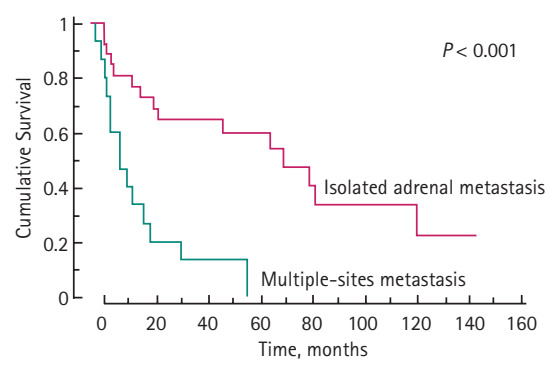

At present, of 14 patients with a solitary metastasis to the adrenal gland, four are alive and free from disease at a mean of 99 months after surgery, two died from causes unrelated to kidney cancer at 3 and 64 months, while the other eight had a relapse at a mean interval of 22.9 (5-57) months after surgery. In three of the last eight patients a second metastasectomy was performed (lung, contralateral adrenal gland, bone) while for the other five no therapy was considered. Two of the patients who had a second metastasectomy (lung and adrenal gland) died from other causes at a mean of 58 months after the second metastasectomy and 110 months after the previous adrenalectomy; the other six patients died at a mean of 15.1 months after adrenalectomy.

The eight patients with multiple metastases treated with curative intent relapsed at a mean of 10.2 (2-35) months after adrenalectomy, and either died or have incurable progression at a mean of 19.4 (2-60) months after adrenalectomy. The remaining 10 patients with diffuse metastatic disease but ineligible for any curative treatment either progressed or died at a mean of 15.4 (2-60) months.

Patients with an isolated metastasis survived significantly longer than those with multiple metastases (overall survival difference, $P=0.008$; disease-free survival difference, $P<0.001)$; there were no significant differences in survival time between patients with multiple metastases who received or did not receive any curative treatments (overall difference, $P=0.816$; disease-free survival, $P=0.606)$.
The 13 patients with a metachronous adrenal metastasis were monitored for a median of 50.5 (12-125) months; in all there were no other apparent relapses at a complete restaging (abdomen-thorax-brain $\mathrm{CT}_{\text {, bone }}$ scan). Only four patients (one still alive and three dead from other causes) had no clinical evidence of further progression of the disease at a mean of 36.8 (12-65) months after adrenalectomy.

The other nine patients had progression at a mean of 33.9 (3-121) months; in three a second metastasectomy was feasible (contralateral kidney and renal fossa, bone, liver) while in the other six no curative treatment was attempted. To date these nine patients are either dead (seven) or have progression (two) at a mean of 57.3 (5-125) months after adrenalectomy, with a mean survival time from relapse after adrenalectomy of 45.8 months in the three surgically treated patients and 5.6 months in the six untreated cases.

The 27 patients whose disease was clinically confined to the adrenal gland (synchronous in 14 and metachronous in 13) had statistically better survival rates than those with diffuse metastasis (overall survival difference, $P=0.01$; disease-free survival, $P<0.001$, Fig. 1). Surgical resection of the adrenal metastasis led to an apparently curative outcome in 10 cases (six with synchronous and four with metachronous metastasis) showing no relapses at a mean of 82.9 months after metastasectomy. These patients had a renal neoplasm without (seven) or with only minimal (three) venous invasion or lymph node involvement.

\section{DISCUSSION}

The incidence of adrenal metastases from RCC is 6-29\% [2-5] in autopsy series, but from clinical diagnosis is less frequent, at 2-10\% [6-9]. In particular, the involvement of the ipsilateral adrenal gland is 19\% in the autopsy and $5.5 \%$ in the surgical series, whereas the contralateral adrenal is involved in up to $11 \%$ of the autopsy series, but with slightly fewer than 60 cases reported as of 2003 [10-12].

In the group of 1179 patients treated for RCC in our department, there was an adrenal metastasis in 45 (incidence 3.7\%). In particular, the metastasis was synchronous in $2.7 \%$, metachronous in $1.0 \%$, ipsilateral in $1.9 \%$, contralateral in $1.5 \%$, and bilateral in $0.3 \%$ of patients.

During the staging of RCC, CT had a high, although not absolute, negative predictive value and a low positive predictive value for the diagnosis of a pathological (for both benign or metastatic) ipsilateral gland: remarkably, false-negative results were more frequent (four) in the group of 10 patients with an isolated unilateral metastasis.

By contrast, there was a high positive predictive capability in diagnosing a pathological contralateral adrenal gland during staging, with only one normal adrenal of 15 removed at the same time as surgery for renal cancer, and during the follow-up, with 13 metastases of 14 . Furthermore, the accuracy of CT for distinguishing between benign and malignant contralateral adrenal nodules has improved recently, using CT protocols to evaluate the wash-out of contrast media [13].

Without considering the presence of distant metastases and taking into account only locoregional staging, synchronous ipsilateral metastases were generally associated with tumours extending to perirenal tissues, to other organs or with lymph node metastases, but in two cases there was a small ( 5 and $6 \mathrm{~cm}$ ) neoplasm confined to the renal capsule (Table 2). From this evidence we think that an adrenal-sparing procedure can be used for organ-confined kidney cancer of $\leq 4 \mathrm{~cm}$, as indirectly confirmed by the absence of adrenal metastases in the 171 patients who had a nephrectomy and adrenalectomy for a neoplasm with the same features. Moreover, none of the 211 patients treated by surgery (nephron-sparing or ablative) with no adrenalectomy for similar tumours developed an ipsilateral metastasis in the long term. Conversely, there were two cases of bilateral metachronous metastasis after an adrenalsparing nephrectomy for a 6-cm pT3a tumour. The lack of involvement of the renal upper pole in 19\% of cases with ipsilateral metastasis, and the incomplete negative predictive capability of CT make the topographic location of tumour and the preoperative ipsilateral adrenal CT findings an unreliable base for selecting adrenal-sparing surgery during nephrectomy. Adrenalectomy could be avoided only during nephrectomy for small renal tumours in nonfunctioning 
kidneys, while an adrenal-sparing procedure for renal tumours of $>4 \mathrm{~cm}$ in diameter is inadvisable, even in the presence of a contralateral adrenal mass. Notably, all the cases of bilateral metastasis had a renal tumour of $>4 \mathrm{~cm}$, and in one case the unilateral adrenal was normal on CT.

The incidence of metachronous adrenal metastasis was, respectively, $0.4 \%$ (three/657) and 3.5\% (10/285) in stage I and III renal tumours, with a wide variability of latency in diagnosis (8-73 months); these values confirm, as recently reported by others [14], that an extended follow-up with $\mathrm{CT}$ is more meaningful for patients with high-stage renal cancer, but also shows how a metastasis can develop in stage I disease. The evidence of metastatic disease confined to the adrenals in all the patients with a metachronous metastasis is probably due to the early diagnosis, but we usually do not routinely use $\mathrm{CT}$, but use abdominal ultrasonography, after a 2-year follow-up with no relapses. These considerations warrant a long-term followup for all patients with RCC using periodic ultrasonography, and suggest the tailored use of CT.

Even if reported data [15] show a greater risk of adrenal insufficiency in patients with RCC, only the 31 patients treated by bilateral adrenalectomy (in one or two steps) chronically required substitution therapy with oral cortisone acetate (25 mg in the morning and $12.5 \mathrm{mg}$ in the afternoon) for glucocorticoid replacement, while mineralocorticoid replacement with oral fludrocortisone (0.1-0.3 mg daily) was used only in patients who had an imbalance in serum electrolytes. There was mostly good tolerance to substitution therapy but careful adjustments are needed during stressful periods to avoid Addisionan crises, as happened in one patient who died from this cause.

The results for the 27 patients shown in Fig. 1 suggest the existence of a preferential pattern of dissemination to the adrenal gland which should outline a 'limited', less aggressive, and therefore surgically curable metastatic disease. However, the relapses experienced by the other patients with an isolated adrenal metastasis, in principle equally eligible for curative surgery, confirm that the true extension of neoplastic disease is still widely underestimated by the available diagnostic tools.
There were no differences in survival between patients with synchronous or metachronous appearance of adrenal metastasis (overall survival difference, $P=0.496$; disease-free survival, $P=0.685$ ), or between those with a uni-, contra- or bilateral metastasis (overall survival difference, $P=0.56$; disease-free survival difference, $P=0.431$ ) even when data were adjusted for the extension of metastatic disease (isolated or disseminated).

In conclusion, in the present 1179 patients with RCC, the incidence of adrenal metastasis was $3.7 \%$, synchronous with the renal tumour in $2.7 \%$ and metachronous in $1.0 \%$. An ipsilateral adrenal-sparing procedure is only safe during surgery for organ-confined RCC of $\leq 4 \mathrm{~cm}$, whereas the location of cancer and the preoperative CT findings cannot fully predict the risk of ipsilateral metastasis. Any suspicious contralateral adrenal mass should be explored because there is a high probability of removing a metastasis.

Oncologically, patients with a solitary adrenal metastasis (synchronous or metachronous) are candidates for adrenal metastasectomy given the good rate of freedom from disease after surgery and the acceptable survival rates when compared with patients who have multiple metastases. These results could justify the indication for surgical resection of adrenal metastasis from RCC but also underline the need for an effective systemic therapy to treat metastatic RCC.

\section{CONFLICT OF INTEREST}

None declared.

\section{REFERENCES}

1 Kovacs G, Akhtar M, Beckwith BJ et al. The Heidelberg classification of renal cell tumours. J Pathol 1997; 183: 131-3

2 Saitoh $H$, Nakayama M, Nakamura K. Distant metastasis of renal adenocarcinoma in nephrectomized cases. J Urol 1982; 127: 1092-5

3 Hellsten S, Berge T, Linell F. Clinically unrecognised renal carcinoma: aspects of tumor morphology, lymphatic and haematogenous metastatic spread. $\mathrm{Br} \mathrm{J}$ Urol 1983; 55: 166-70

4 Bennington JL, Kradjian RM. Distribution of metastasis from renal carcinoma. In Renal Carcinoma, Chapt. 10. Philadelphia: WB Saunders Co., 1999: 1952
5 Hajdu SI, Thomas AG. Renal cell carcinoma at autopsy. J Urol 1967; 97: 978-82

6 Siemer S, Lehmann J, Kamradt J et al. Adrenal metastasis in 1635 patients with renal cell carcinoma: outcome and indication for adrenalectomy. J Uro/ 2004; 171: 2155-9

7 Angervall L, Wahlqvist L. Follow-up and prognosis of renal carcinoma in a series operated by perifascial nephrectomy combined with adrenalectomy and retroperitoneal linfoadenectomy. Eur Urol 1978; 4: 13-7

8 Robey EL, Schellhammer PF. The adrenal gland and renal cell carcinoma: is ipsilateral adrenalectomy a necessary component of radical nephrectomy? J Urol 1986; 135: 453-5

9 Paul R, Mordhorst J, Leyh H, Hartung R. Incidence and outcome of patients with adrenal metastases of renal cell cancer. Urology 2001; 57: 878-82

10 Lau WK, Zincke $\mathrm{H}$, Lohse CM, Cheville JC, Weaver AL, Blute ML. Contralateral adrenal metastasis of renal cell carcinoma: treatment, outcome and a review. BJU Int 2003; 91: 775-9

11 Dieckmann KP, Wullbrand A, Kroizig G. Contralateral adrenal metastasis in renal cell cancer. Scand J Urol Nephrol 1996; 30: $139-43$

12 Kessler OJ, Mukamel E, Weinstein R, Gayer E, Konichezky M, Servadio C. Metachronous renal cell carcinoma metastasis to the contralateral adrenal gland. Urology 1998; 51: 539-43

13 Korobkin M. CT characterisation of adrenal masses: the time has come. Radiology 2000; 217: 629-32

14 Stephenson AJ, Chetner MP, Rourke $\mathrm{K}$ et al. Guidelines for the surveillance of localized renal cell carcinoma based on the patterns of relapse after nephrectomy. J Urol 2004; 172: 58-62

15 Yokoyama H, Tanake M. Incidence of adrenal involvement and assessing adrenal function in patients with renal cell carcinoma: is ipsilateral adrenalectomy indispensable during radical nephrectomy? BJU Int 2005; 95 : 526-9

Correspondence: Alessandro Antonelli, Department of Urology, University of Brescia, Brescia, Italy. e-mail: alxanto@hotmail.com 